\title{
Dilatação e Curetagem na Avaliação do Sangramento Uterino Anormal: Achados Histopatológicos e Relação Custo/Beneficio
}

\author{
Dilatation and Curettage in the Evaluation of Abnormal Uterine Bleeding: \\ Histopathologic Findings and the Cost/Benefit Relation \\ Luiz Augusto Henrique Melki, Marco Aurélio Pinho de Oliveira, Waldyr Tostes Filho, \\ Augusta Maria B. de Assumpção, Hildoberto Carneiro de Oliveira.
}

\section{RESUMO}

Objetivo: avaliar criticamente os achados histopatológicos e a relação custo/beneficio da dilatação e curetagem uterina $(D \& C)$ no rastreio do sangramento uterino anormal (SUA).

Método: análise retrospectiva dos resultados histopatológicos de 542 D\&C praticadas por SUA na Disciplina de Ginecologia da Faculdade de Ciências Médicas da Universidade do Estado do Rio de Janeiro (FCM-UERJ), de janeiro de 1984 a janeiro de 1994. As pacientes foram divididas em dois grupos: Grupo 1 - pacientes com idade igual ou inferior a 50 anos $(n=385)$ e Grupo 2 - pacientes com mais de 50 anos $(n=157)$. Foram excluidos desse estudo os casos de curetagens de urgência. Todas as curetagens foram realizadas sob narcose. O tempo médio de internação foi de três dias. Considerou-se resultado patológico negativo quando o laudo histopatológico mostrou endométrio tipo proliferativo, secretor, atrófico ou iatrogênico. Este último termo referese a endométrio sob possivel influência de medicação hormonal. Considerou-se resultado patológico positivo quando o laudo histopatológico evidenciou algum tipo de lesão.

Resultados: no Grupo 1 encontrou-se resultado patológico negativo em 50,2\% dos casos, resultado patológico positivo em 39,7\% dos casos e material insuficiente para diagnóstico (MIPD) em 10,1\% dos casos. Pólipo endometrial e mioma submucoso foram observados em apenas $5,5 \%$ e 4,4\%, respectivamente. O câncer foi de observação incomum nesse grupo, sendo encontrado o adenocarcinoma do endométrio ( $A C E)$ em apenas 1,3\% dos casos ( $n=5)$, numa relação de 77 D\&C para um ACE. No Grupo 2 observou-se resultado patológico negativo em 38,3\% dos casos, resultado patológico positivo em 38, $1 \%$ dos casos e MIPD em 23,6\% dos casos. Pólipo endometrial e mioma submucoso foram diagnosticados em somente 5,1\% e 0,6\%, respectivamente. Lesões malignas foram encontradas em 12\% dos casos, sendo 9,5\% (15 casos) de ACE, mostrando relação de um ACE para 10 D\&C.

Conclusões: consoante o conhecimento atual sobre a etiopatogenia do SUA este estudo mostrou que a D\&C diagnóstica tradicional tem baixa acurácia na avaliação daquele sangramento $e$ relação custo/beneficio incompativel com a medicina atual. Portanto, não deve ser o exame de primeira escolha. Considerando, contudo, que o ACE foi encontrado em uma de cada $10 \mathrm{D} \& C$ em mulheres com mais de 50 anos com queixa de sangramento uterino, pode-se indicar D\&C com mais liberalidade nesse grupo, uma vez que não se disponha de histeroscopia com biópsia dirigida. Atualmente, a D\&C não tem mais um papel significante no rastreio do SUA como tinha há alguns anos. Entretanto, o procedimento ainda encontra indicação em algumas situações e não pode ser de todo abandonado, devendo sua indicação obedecer a critérios restritos.

PALAVRAS-CHAVE: Sangramento uterino anormal. Histeroscopia. Endométrio: câncer.

Disciplina de Ginecologia da Faculdade de Ciências Médicas da Universidade do Estado do Rio de Janeiro (UERJ), Setor de Endoscopia Ginecológica

Correspondência:

Luiz Augusto Henrique Melki

Av. Prefeito Dulcídio Cardoso, 800; B1 01/ 104 - Barra da Tijuca 22620-311 - Rio de Janeiro - RJ

e-mail: luiz_melki@easyline.com.br
Introdução

O sangramento uterino anormal (SUA) é queixa freqüente e representa aproximadamente $20 \%$ dos motivos de consulta ginecológica ${ }^{1}$. 
Tem etiopatogenia diversa e necessita ser esclarecido com fidedignidade para planejamento terapêutico correto e efetivo. Embora a prática da dilatação cervical e curetagem uterina $(D \& C)$ no diagnóstico do SUA pareça ter diminuído nos últimos anos em função do uso mais freqüente de novos métodos diagnósticos tais como a ultrasonografia e a histeroscopia, observa-se que a curetagem ainda é freqüentemente indicada, seja com objetivos diagnóstico e terapêutico do SUA em mulheres com mais de 35 anos $^{2}$, seja no esclarecimento de espessamento endometrial revelado por ultra-som em mulheres pós-menopáusicas, particularmente se houver sangramento $^{3}$. Críticas à $\mathrm{D} \& \mathrm{C}$ existem há várias décadas, mas apenas na década de 70 um estudo no qual a D\&C foi realizada previamente à histerectomia mostrou que em $60 \%$ dos casos menos de metade da cavidade uterina tinha sido curetada ${ }^{4}$. Esses achados abalaram consideravelmente aquela suposta fidedignidade diagnóstica da D\&C, mas não o suficiente para o abandono do procedimento, tendo em vista a falta de métodos alternativos fidedignos e práticos. Nos anos subseqüentes, a evolução da histeroscopia (invasibilidade mínima) e da ultra-sonografia (nãoinvasiva) possibilitou, respectivamente, a avaliação direta e indireta da cavidade endometrial, sendo ambos os exames realizados ambulatorialmente. Em conseqüência, o papel da tradicional D\&C diagnóstica no rastreio do SUA passou a ser questionável, não só quanto aos aspectos concernentes a custos e riscos, como também quanto aos que tangem a acurácia diagnóstica. Em função dessas controvérsias, o presente trabalho objetiva avaliar não só a qualidade da informação obtida pela D\&C nos casos de SUA como também a relação custo/benefício desse procedimento, para posicioná-la na semiótica ginecológica atual.

\section{Pacientes e Métodos}

Foi feito um estudo retrospectivo analisando-se os laudos anatomopatológicos de 542 D\&C realizadas no Hospital Universitário Pedro Ernesto da Faculdade de Ciências Médicas da Universidade do Estado do Rio de Janeiro (HUPE-UERJ) no período de janeiro de 1984 a janeiro de 1994 . Foram incluídas nesse estudo todas as pacientes encaminhadas pelo Ambulatório de Ginecologia da Disciplina de Ginecologia da Faculdade de Ciências Médicas do Estado do Rio de Janeiro (FCM-UERJ) para avaliação de SUA. Foram excluídas desse estudo aquelas pacientes cure- tadas com urgência. Devido à prevalência do adenocarcinoma do endométrio (ACE) a partir dos 50 anos, as pacientes foram divididas em dois grupos: Grupo 1 - pacientes até 50 anos $(n=385)$ e Grupo 2 - pacientes com mais de 50 anos $(\mathrm{n}=$ 157)

Todas as curetagens foram feitas sob narcose. Todas as pacientes obedeceram à formalidade de se internarem de véspera e terem alta hospitalar, se possivel, no dia seguinte ao procedimento, o que deu, em média, três dias de internação para cada paciente. Considerou-se como resultado patológico negativo o resultado do laudo histopatológico que evidenciou endométrio tipo proliferativo, secretor, atrófico, e endométrio iatrogênico. Este último termo foi cunhado pelos patologistas que examinaram o material para designar endométrio sob possivel influencia de medicação hormonal. Por resultado patológico positivo designaram-se aqueles casos em que o laudo histopatológico mostrou algum tipo de lesão como pólipo endometrial, mioma submucoso, hiperplasia e câncer endometrial.

\section{Resultados}

A Tabela 1 sintetiza os achados histopatológicos referentes ao Grupo 1. Nesse grupo observou-se diagnóstico patológico negativo em $50,2 \%$ dos casos, diagnóstico patológico positivo em $39,7 \%$ dos casos e em $10,1 \%$ dessas $D \& C$ o material foi insuficiente para diagnóstico (MIPD).

Nos casos de diagnóstico patológico positivo, ainda segundo a Tabela 1 , o achado mais freqüente correspondeu às hiperplasias endometriais, encontradas em 21,6\% dos casos. Destas, a hiperplasia simples ocupou o primeiro lugar com $12,7 \%$ dos casos e as hiperplasias cística, adenomatosa e atípica foram encontradas em $3,4 \%, 5 \%$ e $0,5 \%$ dos casos, respectivamente.

Em ordem de freqüência, após as hiperplasias, observou-se o achado anatomopatológico de pólipo endometrial e mioma submucoso com taxas respectivas de $5,5 \%$ e $4,4 \%$ dos casos.

A malignidade ocorreu em apenas 2,8\% dos casos no Grupo 1. Tanto o ACE quanto o carcinoma epidermóide (colo do útero) foram encontrados em taxas de 1,3\% dos casos, respectivamente. Observou-se, também, nesse grupo, um caso de sarcoma $(0,2 \%)$.

Ainda consoante a Tabela 1 , no subgrupo rotulado como Miscelânea, verificou-se predomínio de achados anatomopatológicos (3,3\%) compativeis com prenhez abortada ou ectópica. 
Tabela 1 - Diagnóstico histopatológico em 385 D\&C para avaliação de SUA em pacientes $=50$ anos.

\begin{tabular}{|c|c|c|c|}
\hline Histopatologia & $\mathrm{n}$ & $\%$ & Total \\
\hline Endométrio proliferativo e secretor & 138 & 35,9 & \\
\hline Endométrio atrófico & 13 & 3,4 & \\
\hline Endométrio iatrogênico & 42 & 10,9 & $50,2 \%$ \\
\hline Pólipo endometrial & 21 & 5,5 & \\
\hline Mioma submucoso & 17 & 4,4 & \\
\hline \multicolumn{4}{|l|}{ Hiperplasia } \\
\hline Simples & 49 & 12,7 & \\
\hline Cística & 13 & 3,4 & \\
\hline Adenomatosa & 19 & 5,0 & \\
\hline Atípica & 2 & 0,5 & \\
\hline \multicolumn{4}{|l|}{ Malignidade } \\
\hline ACE & 5 & 1,3 & \\
\hline Carcinoma epidermóide (colo) & 5 & 1,3 & \\
\hline Sarcoma & 1 & 0,2 & \\
\hline \multicolumn{4}{|l|}{ Miscelânea } \\
\hline Reação deciduóide & 6 & 1,6 & \\
\hline Endometrite aguda & 5 & 1,3 & \\
\hline Reação de Arias-Stella & 4 & 1,0 & \\
\hline Descamação irregular do endométrio & 3 & 0,8 & \\
\hline Vilosidades hialinizadas & 2 & 0,5 & \\
\hline Hiperplasia gestacional & 1 & 0,2 & $39,7 \%$ \\
\hline Material insuficiente & 39 & 10,1 & $10,1 \%$ \\
\hline Total & 385 & 100 & \\
\hline
\end{tabular}

A Tabela 2 resume os achados histopatológicos do Grupo 2, no qual se observou resultado patológico negativo em $38,3 \%$, resultado patológico positivo em $38,1 \%$ do casos e MIPD em $23,6 \%$ dos casos, somando-se, nesse grupo, total de $61,9 \%$ de casos inconclusivos quanto à fonte do SUA. O achado de endométrio atrófico foi observado em $22,3 \%$ dos casos.

Em relação aos achados patológicos positivos, predominaram as hiperplasias endometriais, somando 19,2\% dos casos. Destas, a hiperplasia simples foi a mais freqüente $(10,9 \%)$, a hiperplasia cística foi observada em $4,5 \%$ dos casos e a adenomatosa e a atípica foram encontradas em taxas iguais de 1,9\%.

A malignidade ocupou o segundo lugar após os achados de hiperplasias endometriais. $\mathrm{O}$ ACE foi encontrado em $(9,5 \%) 15$ casos. O carcinoma epidermóide do colo do útero e o sarcoma foram diagnosticados em $1,9 \%$ e $0,6 \%$, respectivamente. O pólipo endometrial e o mioma submucoso foram relatados em $5,1 \%$ e $0,6 \%$ dos casos, respectivamente.
Tabela 2 - Diagnóstico histopatológico em 157 D\&C para avaliação do SUA em pacientes com mais de 50 anos

\begin{tabular}{lrrr}
\hline Histopatologia & $\mathbf{n}$ & \multicolumn{1}{c}{$\%$} & Total \\
\hline Endométrio proliferativo e secretor & 14 & 9 & \\
Endométrio atrófico & 35 & 22,3 & \\
Endométrio iatrogênico & 11 & 7,0 & $38,3 \%$ \\
Pólipo endometrial & 8 & 5,1 & \\
Mioma submucoso & 1 & 0,6 & \\
Hiperplasia & & & \\
Simples & 17 & 10,9 & \\
Cística & 7 & 4,5 & \\
Adenomatosa & 3 & 1,9 & \\
Atípica & 3 & 1,9 & \\
Malignidade & & & \\
ACE & 15 & 9,5 & \\
Epidermóide (colo) & 3 & 1,9 & \\
Sarcoma & 1 & 0,6 & \\
Miscelânea & 2 & 1,2 & $38,1 \%$ \\
Material insuficiente & 37 & 23,6 & $23,6 \%$ \\
Total & 157 & 100 & \\
\hline
\end{tabular}

O resultado final da análise dos dois grupos mostra que no tocante ao diagnóstico, em $61 \%$ dos casos não se registrou resultado patológico e o reflexo dessas 542 D\&C sobre a internação hospitalar indica que o hospital arcou com 987 dias de internação em 329 D\&C em que o resultado patológico foi negativo. O tempo médio de internação para cada paciente foi de três dias.

\section{Discussão}

À luz da análise dos achados histopatológicos de curetagens indicadas por SUA, torna-se evidente que esse procedimento tem indicações restritas nos dias atuais, embora ainda tenha valor em umas poucas situações.

Os laudos de nossa casuística facilmente confirmam o que foi dito acima. Em ambos os grupos o raspado endometrial mostrou unicamente endométrio fisiológico em taxas bastante expressivas, deixando o médico em dúvida quanto ao real diagnóstico etiopatogênico do SUA. No Grupo 1 essas taxas chegaram a representar metade das curetagens realizadas. No Grupo 2 esses índices foram menores, mas, mesmo assim, ultrapassaram um terço $(38,3 \%)$ das D\&C praticadas. Essas altas taxas de resultados anatomopatológicos mostrando apenas tipo fisiológico ou iatrogênico de endomé- 
trio em D\&C por SUA e, em conseqüência, não permitindo diagnóstico algum, é fato já relatado em outras estatísticas. Nos dados de Bertuol et al. ${ }^{5}$ compreendendo $1032 \mathrm{D} \& \mathrm{C}$, o diagnóstico de endométrio proliferativo e secretor correspondeu a $61,2 \%$ dos casos. Da mesma forma, das $278 \mathrm{D} \& \mathrm{C}$ relatadas por Neis et al. ${ }^{6}$ $74,5 \%$ dos laudos corresponderam a diagnóstico histológico de endométrio proliferativo, secretor, não-funcional e atrófico. Embora muitas vezes esses diagnósticos traduzam a real situação, e o caso possa ser corretamente rotulado como de sangramento uterino disfuncional, o diagnostico patológico negativo à $\mathrm{D} \& \mathrm{C}$ não significa inexistência de lesão intracavitáriaa ${ }^{7-9}$. Sabemos que pólipos endometriais e miomas submucosos, por exemplo, podem coexistir com qualquer tipo de endométrio e que a curetagem pode não detectar a lesão, retirando apenas endométrio circundante ${ }^{6}$. Uma possivel explicação para esses achados parece residir na ampla mobilidade dos pólipos pediculados, fazendo-os fugidios à cureta e na consistência firme e fibroelástica dos miomas, dificultando a tomada de biopsias ou a sua retirada pela curetagem. Mediante histeroscopia adjunta à $\mathrm{D} \& \mathrm{C}$ pode-se comprovar que muitos pólipos escapam à cureta $^{10}$ e que uma única curetagem retira menos de um terço de pequenos pólipos ou miomas submucosos ${ }^{11}$. Por outro lado, essas lesões são freqüentemente encontradas ao exame histeroscópico quando a D\&C fracassa no tratamento do $\mathrm{SUA}^{8,9}$.

Outro aspecto que merece comentários é quanto ao laudo diagnóstico de MIPD. De modo geral esse achado indica endométrio senil que não fornece material à raspagem. Todavia, esse diagnóstico também pode aparecer em D\&C praticadas em mulheres na pré-menopausa ${ }^{5,9}$. É achado que se apresenta com freqüência variável segundo os diversos autores. No presente trabalho foi observado em $23,6 \%$ no grupo $>50$ anos; nos dados de Choo et al. ${ }^{12}$ em $42 \%$ (pacientes pós-menopáusicas), e na estatística de Spiewankiewicz et al. ${ }^{9}$ em 46,1\%. Assim, é previsivel que no sangramento uterino na pós-menopausa alto percentual de D\&C (podendo-se chegar até a quase $50 \%$ ) trará pouca informação, e portanto, os custos e os riscos desse procedimento em face dos benefícios duvidosos só se justificam se este método for o único disponivel.

Sabemos que pelo exame histeroscópico a etiologia mais freqüente do sangramento uterino na pós-menopausa é de atrofia endometrial ${ }^{12,13}$. Curiosamente, na casuística relatada por Choo et $a .^{12}$ a histeroscopia confirmou atrofia do endométrio nos $42 \%$ em que a $D \& C$ não retirou material, mas em um estudo de nossa autoria ${ }^{13}$ verificamos que em sete casos de sangramento uterino da pós-menopausa em que o laudo da biópsia endometrial dirigida foi de MIPD, o exame histeroscópico mostrou alguma lesão em 43\% dos casos, sendo uma delas um ACE focal.

Tendo em vista esses achados, reiteramos a importância do exame histeroscópico no rastreio do SUA, devendo ser indicado mesmo que curetagem prévia não forneça material para análise histopatológica. No que concerne ao diagnóstico das hiperplasias endometriais, principalmente as de alto risco, a D\&C parece ser efetiva, pois o diagnóstico pela cureta ${ }^{14-16}$ mostra taxas semelhantes àquelas encontradas pela histeroscopia ${ }^{17,18}$. A maioria dessas lesões acomete difusamente a mucosa endometrial, o que as torna de fácil acesso à cureta. Contudo, quando se apresentam como lesões focais, o risco de não serem diagnosticadas pela curetagem é real. Stock e Kanbour ${ }^{4}$ demonstraram definitivamente que a capacidade exploratória da cavidade uterina pela D\&C não era tão completa quanto se imaginava. Em 50 D\&C praticadas por 24 obstetras-ginecologistas, esses autores verificaram na peça de histerectomia que em 16\% dos espécimes menos que um quarto da cavidade tinha sido curetada, em $60 \%$ menos que metade da cavidade tinha sido curetada e em $84 \%$ menos de três quartos da cavidade endometrial tinha sido efetivamente curetada.

Valle ${ }^{19}$ comparou a sensibilidade da biopsia sob visão histeroscópica com $\mathrm{D} \& \mathrm{C}$ no rastreio do ACE e das hiperplasias endometriais. Verificou que, quando as lesões comprometiam larga porção da cavidade endometrial, todas foram diagnosticadas pela curetagem. No entanto, de três pacientes com ACE focal apenas um caso foi detectado pela D\&C; de 13 pacientes com hiperplasia focal do endométrio apenas duas tiveram diagnóstico pela curetagem uterina.

Outro aspecto que merece análise é a acurácia da $\mathrm{D} \& \mathrm{C}$ no diagnóstico de pólipo endometrial e mioma submucoso. E essas são as mais freqüentes dentre as lesões associadas ao SUA $^{7-}$ $9,11,13$. Num estudo histeroscópico em mulheres com SUA na peri e pós-menopausa, miomas submucosos e pólipos endometriais, em conjunto, somaram $37,8 \%$ e $35,8 \%$ dos casos, para os respectivos grupos ${ }^{20}$. Em verdade, a D\&C é procedimento de muito pouco préstimo em relação aos miomas submucosos e apresenta alta taxa de insucesso no diagnóstico dos pólipos endometriais, conforme pode ser deduzido pela análise da Tabela 3, na qual se confrontam as taxas de diagnóstico daquelas lesões por histeroscopia e por curetagem. Em relação aos miomas 
submucosos parece-nos duvidosa a eficácia da D\&C em diagnosticá-los, e muitas estatíscas de resultados de curetagens para rastreio do SUA nem os menciona em seus dados. A sensação táctil de irregularidade da cavidade endometrial à cureta pode supor a existência de mioma submucoso, mas é critério absolutamente subjetivo, portanto, pouco fidedigno. O que pode acontecer é que curetagem mais enérgica remova fragmento do mioma (o que parece não ser freqüente) e, destarte, o patologista pode fazer o diagnóstico da lesão.

Tabela 3 - Taxas (\%) de diagnósticos de pólipo endometrial e mioma submucoso por D\&C e por histeroscopia no sangramento uterino anormal.

\begin{tabular}{lrcc}
\hline Autor & $\mathbf{n}$ & $\begin{array}{c}\text { Pólipo } \\
\text { endometrial }\end{array}$ & $\begin{array}{c}\text { Mioma } \\
\text { submucoso }\end{array}$ \\
\hline D \& C & 1032 & 2,5 & - \\
Bertuol et al. & 278 & 3,6 & - \\
Neis et al. & & & \\
Spiewankiewicz et al. $^{9}$ & 202 & - & - \\
Valle $^{19}$ & 419 & 3,5 & 0,0 \\
Presente trabalho & 542 & 5,3 & 3,3 \\
Histeroscopia & & & \\
Spiewankiewicz et al. $^{9}$ & 202 & 18,8 & 10,4 \\
Valle $^{19}$ & 419 & 39,4 & 16,2 \\
Melki \& Tostes Filho $^{20}$ & 809 & 12,4 & 21,2 \\
Melki $^{13}$ & 82 & 10,4 & 15,0 \\
Loffer $^{29}$ & 187 & 16,0 & 11,2 \\
\hline
\end{tabular}

-: dado não-mencionado

A relação custo/benefício da $\mathrm{D} \& \mathrm{C}$ no rastreio do SUA no menacme ou do sangramento uterino na pós-menopausa mostrou-se altíssima e proibitiva nos dias atuais. Na estatística do presente trabalho a vasta maioria das curetagens $(71 \%)$ foi realizada em pacientes com 50 anos ou menos, faixa etária em que o SUA apresenta-se mais freqüentemente e que a hiperplasia atípica e o $\mathrm{ACE}$ - lesões que a $\mathrm{D} \& \mathrm{C}$ diagnostica com mais eficiência - ocorreram em apenas $0,5 \%$ e $1,3 \%$ dos casos, respectivamente. Em outras palavras, registraram-se 192 D\&C para uma hiperplasia atipica e $77 \mathrm{D} \& \mathrm{C}$ para um ACE na citada faixa etária. Registre-se, ainda, que nesse grupo $60,3 \%$ dos casos ficaram sem diagnóstico quanto à possivel fonte de sangramento, representando relação custo/benefício não mais justificável atualmente.

Em relação às pacientes $>50$ anos, a relação custo/benefício também se mostrou alta- mente desfavorável para os dias atuais tendo em vista a disponibilidade de modernos exames ultra-sonográficos ou endoscópicos, de realização ambulatorial. Nesse grupo, segundo nossa estatísca, $61,9 \%$ das D\&C não trouxeram esclarecimento quanto ao motivo do sangramento. Destaque-se que $23,6 \%$ das curetagens não deram material para análise histológica, o que é esperado para a faixa etária, mas eleva muito a relação custo/beneficio. Todavia, a preocupante taxa de 10 D\&C para o achado de um ACE parece, a nosso ver, suavizar um pouco aquela relação desfavorável e justificar propedêutica mais abrangente no rastreio do sangramento uterino em mulheres de 50 anos ou mais, caso não se disponha do exame histeroscópico.

Vale a pena lembrar, entretanto, que a biopsia endometrial sem histeroscopia tem boa sensibilidade no diagnóstico do ACE: 86,2\% (cureta Vabra) segundo Vuopala ${ }^{21}$. Portanto, a vasta maioria dos ACE pode ser diagnosticada com simples biopsia endometrial, procedimento de ambulatório na maioria das vezes, e que deve ser levado em conta quando se cogitar da D\&C como primeiro exame para esclarecer sangramento uterino em mulheres na quinta década da vida em diante. É claro que sendo a biopsia negativa, faz-se necessário o esclarecimento por outros métodos, uma vez que até a curetagem convencional dá resultado falso-negativo de $8 \%$ em média no diagnóstico do $\mathrm{ACE}$, principalmente quando a lesão se localiza em pólipos ou na região cornual da cavidade uterina ${ }^{21}$.

$\mathrm{O}$ aviso quanto aos altos custos da D\&C vem de longa data. Grimes ${ }^{22}$ chamou a atenção para o fato de que os alegados benefícios da curetagem ainda não tinham sido comprovados e, só nos Estados Unidos, se gastava aproximadamente um bilhão de dólares com os custos anuais das D\&C. Propôs, ainda, a necessidade de substituí-la, pelo menos em grande percentual, por procedimento mais simples, seguro e mais conveniente, o que, na opinião do autor, seria a aspiração endometrial pelo dispositivo Vabra ("Vabra aspiration"), de prática ambulatorial. Embora despendêssemos esforços para avaliar os custos das D\&C da nossa estatística, não logramos êxito total dada à complexidade de um hospital universitário. De qualquer forma, verificamos que no caso específico do HUPE-UERJ, as 542 D\&C custaram o equivalente a 1626 dias de internação. Desse custo, o equivalente a 987 dias de internação (representando as 329 curetagens sem diagnóstico etiopatogênico do SUA) foi pouco frutífero. Tendo em consideração que aproximadamente $60 \%$ das D\&C praticadas por SUA ficarão sem diagnóstico etio- 
patogênico preciso, fica claro que os beneficios desse procedimento conta pouco nos dias atuais em que outros exames, menos dispendiosos e mais fidedignos, estão à disposição do médico no rastreio do SUA.

Não se trata, contudo, de se abolir inteiramente a D\&C do armamentário do ginecologista, mas, sem dúvida, temos que indicá-la sob critérios rigorosos sob pena de aumento excessivo de riscos e custos médico-hospitalares diante de beneficios bastante duvidosos. Destarte, a nosso ver caberia indicação de curetagem em algumas situações. Quando a biópsia do endométrio mostrar hiperplasia adenomatosa, pois o carcinoma oculto pode coexistir com esse tipo de hiperplasia em cerca de $30 \%$ dos $\operatorname{casos}^{23}$; da mesma forma, o achado de hiperplasia atípica à biopsia merece estudo de maior quantidade de endométrio, uma vez que a $\mathrm{D} \& \mathrm{C}$ tem mais acurácia do que a biópsia na avaliação do grau de diferenciação do câncer endometrial ${ }^{24}$. Nos casos em que há discordância entre impressão histeroscópica e biópsia endometrial, principalmente quando o diagnóstico histeroscópico sugerir lesão grave; nestas circunstâncias, a histeroscopia com ou sem biopsia sob controle visual pode ser repetida sob narcose e complementada por curetagem que possibilitará maior quantidade de endométrio para exame. A curetagem deve ser indicada como exame adjunto à exérese histeroscópica de pólipos em pacientes pós-menopáusicas com endométrio hiperplástico e quando não se dispuser de histeroscopia operatória e o exame histeroscópico e/ou ultra-sonográfico ou a histero-sonografia sugerir lesão compativel com pequeno pólipo endometrial em pacientes sintomáticas.

Só há pouco menos de duas décadas aperfeiçoaram-se métodos semióticos que podem competir vantajosamente com a D\&C. Dentre esses destacam-se a ultra-sonografia e histeroscopia, esta, atualmente, de prática ambulatorial. A histero-sonografia vaginal combinada com biópsia do endométrio parece ser confiável na avaliação de mulheres pós-menopáusicas com sangramento uterino ${ }^{25}$. Ademais, esse exame tem sido empregado com freqüência na distinção entre pacientes que apresentam endométrio de espessura mínima e sangram em decorrência de anovulação da perimenopausa ou da atrofia da pós-menopausa, daquelas que apresentam espessamento endometrial e que necessitam de mais investigação ${ }^{26}$. Porém, não permitem biópsia dirigida e não avaliam sinais mais sutis como vascularização e friabilidade da mucosa endometrial.

Por possibilitar visão direta do canal cervical e da cavidade uterina e permitir biópsia dirigida ou sob controle visual, não resta dúvida que a histeroscopia é, no momento, o exame mais fidedigno na avaliação das condições patológicas da cavidade endometrial, substituindo com ampla vantagem a $\mathrm{D} \& \mathrm{C}$ diagnóstica. Além dos riscos e custos muito menores do método histeroscópico, estudos comparativos entre histeroscopia e D\&C mostraram que a primeira informa bem mais que a segunda ${ }^{27}$, e que a sensibilidade da histeroscopia é maior (98\%) do que a da D\&C $(65 \%)^{28}$ no rastreio do SUA. Ademais, o exame histeroscópico permite diagnosticar lesões benignas com grau de certeza bastante razoável e torna possivel o planejamento terapêutico destas, tarefa que a $\mathrm{D} \& \mathrm{C}$ não pode realizar a contento.

As altas taxas de diagnósticos inconclusivos nos casos de SUA avaliados pela D\&C (mais de $50 \%$ em nossos achados) e a baixa acurácia do método no diagnóstico de pólipos e miomas submucosos elevam extraordinariamente a relação custo/benefício, tornando-a incompatível com a realidade médica atual e deixando-a em inferioridade frente aos métodos modernos de investigação da cavidade uterina. Em conseqüência, não detem mais a D\&C o lugar importante que ocupava há alguns anos na semiótica ginecológica, mas ainda não pode ser de todo abandonada, subordinando suas indicações a critérios restritos.

\section{SUMMARY}

Purpose: to critically evaluate the histopathologic findings and the cost/benefit relation of dilatation and uterine curettage $(D \& C)$ in the evaluation of the abnormal uterine bleeding (AUB).

Method: retrospective analysis of the histopathological findings in $542 D \& C$ performed for AUB in the Department of Gynecology of the Faculdade de Ciencias Médicas da Universidade do Estado do Rio de Janeiro (FCM-UERJ), between January 1984 and January 1994. The patients were divided into two groups: Group 1 - patients $\leq 50$ years $(385 D \& C)$ and Group 2 - patients $>50$ years $(157 D \& C)$. Cases of urgency curettage were excluded from the study. All the curettages were accomplished under narcosis. The mean hospitalization lenght was three days. A histopathological finding of proliferative, secretory, atrophic or iatrogenic type endometrium was considered a negative pathological result. The term iatrogenic refers to the endometrium under possible influence of hormonal medication. When the histopathological finding evidenced 
some lesion, this was considered a positive pathological result.

Results: in Group 1 there was a negative pathological result in $50.2 \%$ of the cases, positive pathological result in $39.7 \%$ of the cases, and insufficient material for diagnosis (IMD) in $10.1 \%$ of the cases. Endometrial polyp and submucosal leiomyoma were found in only $5.5 \%$ and $4.4 \%$, respectively. Cancer was an uncommon observation in that group, endometrial adenocarcinoma (EAC) (five cases) being found in only $1.3 \%$ of the cases, in a relation of $77 D \& C$ to one EAC. In Group 2, a negative pathological result was observed in $38.3 \%$ of the cases, positive pathological result in $38.1 \%$ of the cases and IMD in $23.6 \%$ of the cases. Endometrial polyp and submucosal leiomyoma were found only in $5.1 \%$ and $0.6 \%$, respectively. Malignant lesions were found in $12 \%$ of the cases EAC being 9.5\% (15 cases), showing a relation of one EAC to $10 \mathrm{D \& C}$.

Conclusions: according to the current knowledge on the etiology of $A U B$, this study showed that traditional diagnostic D\&C has low accuracy in the evaluation of $A U B$ and a cost/benefit relation incompatible with current medicine. Therefore, it should not be the examination of first choice. Considering, however, that EAC was found in one of each $10 \mathrm{D \& C}$ in women $>50$ years with a complaint of uterine bleeding, D\&C can be indicated with more liberality in that group, if hysteroscopy with directed biopsy is not available. Nowadays, D\&C does not play such a significant a role in the diagnosis of $A U B$ as it did some years ago. However, the procedure is still indicated in some situations and it cannot be abandoned, and its indication should obey restricted criteria.

KEY WORDS: Curettage. Metrorrhagia. Hysteroscopy. Endometrium: cancer.

\section{Referências}

1. Jennings JC. Abnormal uterine bleeding. Med Clin North Am 1995; 79:1357-76.

2. Kim MH. Sangramento uterino disfuncional. In: Copeland LJ, editor. Tratado de Ginecologia. $1^{\text {a }}$ ed. Rio de Janeiro: Guanabara Koogan; 1996. p.377.

3. Fleischer AC, Entman SS. Avaliação ultrasonográfica do útero e distúrbios relacionados. In: Fleischer AC, Maning FA, Jeanty P, Romero $\mathrm{R}$, editores. Ultra-sonografia em Obstetricia e Ginecologia: princípios e prática. $1^{\text {a }}$ ed. Rio de Janeiro: Revinter; 2000. p.845.

4. Stock RJ, Kanbour A. Prehysterectomy curettage. Obstet Gynecol 1975; 45:537-41.

5. Bertuol M, Frasson AL, Silveira GPG. Sangramento uterino anormal quando investigar? GO Atual 1993; 6:33-40.
6. Neis KJ, Brandner P, Hepp H. Hysteroscopy: textbook and atlas. $1^{\text {st }}$ ed. Stuttgart: Thieme Verlag; 1994. p.79.

7. Word B, Gravlee C, Gilder WL. The fallacy of simple uterine curettage. Obstet Gynecol 1958; 12:6428.

8. Brooks PG, Serden SP. Hysteroscopic findings after unsuccessful dilatation and curettage for abnormal uterine bleeding. Am J Obstet Gynecol 1988; 158:1354-7.

9. Spiewankiewicz B, Stelmachów J, Sawicki W, Kietlinska Z. Hysteroscopy with selective endometrial sampling after unsuccessful dilatation and curettage in diagnosis of symptomatic endometrial cancer and endometrial hyperplasias. Eur J Gynaecol Oncol 1995; 16:26-9.

10.Burnett JE. Hysteroscopy-controlled curettage for endometrial polyps. Obstet Gynecol 1964; 24:62135.

11. Goldfarb HA. D\&C results improved by hysteroscopy. N J Med 1989; 86:277-9.

12. Choo YC, Mak KC, Hsu C, Wong TS, Ma HK. Postmenopausal uterine bleeding of nonorganic cause. Obstet Gynecol 1985; 66:225-8.

13. Melki LAH. Histeroscopia diagnóstica em pacientes de 50 anos ou mais com hemorragia uterina anormal [tese]. Rio de Janeiro: Universidade Estadual do Rio de Janeiro; 1992.

14.Wentz WB. Treatment of persistent endometrial hyperplasia with progestins. Am J Obstet Gynecol 1966; 96:999-1004.

15. Sherman AI, Brown S. Precursors of endometrial carcinoma. Am J Obstet Gynecol 1979; 135:94756.

16. Bastos AC. Hiperplasia endometrial. In: Noções de ginecologia. 8 ${ }^{a}$ ed. São Paulo: Atheneu; 1991. p.276.

17.Tantini C, Mencaglia L. Diagnosis of endometrial cancer and its precursor using hysteroscopy. Gynaecol Endosc 1994; 3 Suppl:28.

18. Melki LAH, Tostes Filho W, Lemos PAP, Oliveira MAP. Histeroscopia ambulatorial e biópsia dirigida no diagnóstico das hiperplasias endometriais de alto risco. Med HUPE-UERJ 1995; 14 Suppl:111.

19.Valle RF. Hysteroscopic evaluation of patients with abnormal uterine bleeding. Surg Gynecol Obstet 1981; 153:521-6.

20.Melki LAH, Tostes Filho W. Histeroscopia panorâmica: atlas e texto. $1^{\text {a }}$ ed. Rio de Janeiro: EPUC; 1992. p. 61-2.

21.Vuopala S. Diagnostic accuracy and clinical applicability of cytological and histological methods for investigating endometrial carcinoma. Acta Obstet Gynecol Scand Suppl 1977; (70):21. 
22.Grimes DA. Diagnostic dilatation and curettage: a reappraisal. Am J Obstet Gynecol 1982; 142:1-6.

23. Champion MJ, Reid R. Screening for gynecologic cancer. Obstet Gynecol Clin North Am 1990; 17:695-727.

24.Larson DM, Johnson KK, Broste SK, Krawisz BR, Kresl JJ. Comparison of D\&C and office endometrial biopsy in predicting final histopathologic grade in endometrial cancer. Obstet Gynecol 1995; 86:38-42.

25. O'Connell LP, Fries MH, Zeringue E, Brehm W. Triage of postmenopausal bleeding: A comparison of endometrial biopsy and transvaginal sonohysterography versus fractional curettage with hysteroscopy. Am J Obstet Gynecol 1998; 178:958-61.

26.Lurain JR. Uterine cancer. In: Berek JS, Adashi EY, Hillard PA, editors. Novak's gynecology.12a ed. Baltimore: Williams \& Wilkins; 1996. p.1063.

27.Gimpelson RJ, Rappold HO. A comparative study between panoramic hysteroscopy with directed biopsies and dilatation and curettage. Am J Obstet Gynecol 1988; 158:489-92.

28.Loffer FD. Hysteroscopy with selective endometrial sampling compared with $\mathrm{D} \& \mathrm{C}$ for abnormal uterine bleeding: the value of a negative hysteroscopic view. Obstet Gynecol 1989; 73:1620. 